intervention for physicians' burnout. These results suggest that strategies including forgiveness training aimed at decreasing WB while increasing job satisfaction among physicians warrant further exploration. Funding Acknowledgements: no funding

43

\section{Tinnitus as an Aura for Sleep Paralysis}

Luvleen Shergill, MS4'; Jasir Nayati, MS4'ㄹ Reshma Nair, $\mathrm{MS4}^{3}$; and Alan R. Hirsch, $M D^{4}$

${ }^{1}$ Aureus University School of Medicine, Oranjestad, Aruba

${ }^{2}$ St. James School of Medicine, Anguilla

${ }^{3}$ American University of Integrative Sciences, Barbados

${ }^{4}$ Smell and Taste Treatment and Research Foundation, Chicago, IL

ABSTRACT: Objective: To understand that tinnitus may be an aura for sleep paralysis.

BACKGROUND: Sleep paralysis is a transient-paralysis which occurs during awakening or falling asleep (Wilson, 1928). Those affected experience symptoms including visual, auditory, and haptic hallucinations, voluntary motor paralysis with intact ocular and respiratory motor movements, and diffuse or localized paresthesias. Sleep paralysis associated with tinnitus as an aura, has not heretofore been described.

METHODS: A 34 year-old, right-handed female presented with a 13 year history of sleep paralysis. One month prior, she began to notice tinnitus prior to the onset of sleep paralysis. The tinnitus was bilateral, high-pitched, with a volume intensity of $5 / 10$, lasting seven seconds prior to sleep initiation. She denied hearing loss, vertigo, dizziness, cataplexy, deja vu and jamais vu. After termination of tinnitus, she experienced paresthesia, "like at a dentist's office" radiating from her posterior neck, to her tongue and down to her toes. She described seeing a white-shadowy male figure moving around her room, lasting seven seconds. Accompanied by a masculine "ahh" sound, lasting for three seconds. The sleep paralysis occurred after these events, lasting up to eight hours, or until her husband wakes her.

RESULTS: Abnormalities in Physical Examination: General Examination: right arm hemangioma 4 by $5 \mathrm{~cm}$. Reflexes: absent bilateral brachioradialis, $1+$ bilateral quadriceps femoris and bilateral Achilles tendon. Neuropsychiatric Examination: Calibrated Finger Rub Auditory Screening Test: faint $70 \mathrm{AU}$ (normal).

DISCUSSION: Tinnitus has been described as an aura for migraines (Schankin, 2014), temporal lobe epilepsy (TLE)
(Florindo, 2006), and narcolepsy-cataplexy (Marco, 1978). These epochs may represent amigranous migraines, which initially present with tinnitus that occurs both during the day and night, forcing the patient to be partially awoken at night with induction of the sleep paralysis sequence. It would be worthwhile to query those with narcolepsy or sleep paralysis if tinnitus precedes the event.

44

Real World Effectiveness: A 6-month Naturalistic Follow-up Study of Schizophrenia Patients After Switching to Aripiprazole Monohydrate (AOM) Treatment

Marcos Gómez-Revuelta, MD-PhD ${ }^{1,2}$; José María Pelayo-Terán, MD-PhD ${ }^{1,2,3}$; María Juncal-Ruiz, $M D^{1,2}$, María Fernández-Rodríguez, $M D^{1,2}$; Javier VázquezBourgon, MD-PhD ${ }^{1,2}$; Paula Suárez-Pinilla, $\mathrm{MD}^{1,2}$; and Oriol Porta-Olivares, $M D^{1,2}$

${ }^{1}$ University Hospital Marqués de Valdecilla, IDIVAL, Department of Psychiatry, School of Medicine, University of Cantabria, Santander, Spain

${ }^{2}$ CIBERSAM, Centro Investigación Biomédica en Red Salud Mental, Madrid, Spain

${ }^{3}$ Unidad de Gestión Clínica de Psiquiatría y Salud Mental. Hospital El Bierzo. Servicio de Salud de Castilla y León (SACYL), Ponferrada (León), Spain

ABSTRACT: Rationale: Long-acting injectable antipsychotic therapies may offer benefits over oral antipsychotics in patients with schizophrenia. However, there is still a lack of real-world studies assessing the effectiveness of these therapies.

OBJECTIVE: This study aimed to explore the safety, tolerability, and treatment response of aripiprazole monohydrate (AOM) once monthly in non-acute but symptomatic adult patients switched from previous therapy with frequently used oral or injectable atypical antipsychotics.

METHODS: This was a post hoc analysis of a prospective, interventional, single-arm, open-label, 6-month study.

RESULTS: The patients $(\mathrm{N}=54)$ were switched to aripiprazole monohydrate once-monthly (AOM) from daily oral treatment or monthly injectable treatment with either aripiprazole $(n=25)$, olanzapine $(n=7)$, paliperidone extended-release (PP1M) $(n=10)$, quetiapine $(\mathrm{n}=4)$, or risperidone $(\mathrm{n}=8)$. In all groups, mean Positive and Negative Syndrome Scale total $(\mathrm{p}=0.0001)$ and Clinical Global Impression-Severity scores improved significantly $(\mathrm{p}=0.0001)$. A reduction of $\geq 50 \%$ reduction of BPRS total-score and a CGI 